\title{
CORRIGENDA
}

\section{Imaging dopamine transmission in the frontal cortex: a simultaneous microdialysis and $\left[{ }^{11} \mathrm{C}\right] \mathrm{FLB} 457$ PET study}

R Narendran, HP Jedema, BJ Lopresti, NS Mason, K Gurnsey, J Ruszkiewicz, C-M Chen, L Deuitch, WG Frankle and CW Bradberry

Molecular Psychiatry (2014) 19, 399; doi:10.1038/mp.2013.52; published online 23 April 2013

Correction to: Molecular Psychiatry advance online publication, 26 February 2013; doi:10.1038/mp.2013.9

Following publication of the above article, the authors noticed an omission in the Disclaimer section and several errors in Table 3. The complete Disclaimer appears below:

The content is solely the responsibility of the authors and does not necessarily represent the official views of the NIDA, the NIAAA, the National Institutes of Health, or the Veterans Administration (VA).
In Table 3, some of the values in the far-right column of the section for the $0.5-\mathrm{mg} \mathrm{kg}^{-1}$ dose did not appear in the correct row. The corrected table appears below:

Table 3. Amphetamine-induced change in $\left[{ }^{11} \mathrm{C}\right] \mathrm{FLB} 457 \mathrm{BP}$ ND and dialysate $\mathrm{DA}$ in frontal cortex

\begin{tabular}{|c|c|c|c|c|c|c|c|}
\hline $0.3 \mathrm{mg} \mathrm{kg}^{-1}$ & A & 0.85 & 0.73 & -14 & 0.53 & 837 & 671 \\
\hline \multirow[t]{4}{*}{ MD: $n=4$ experiments $/ 4$ animals } & C & 0.97 & 0.86 & -11 & 0.27 & 1100 & 924 \\
\hline & D & 0.89 & 0.89 & +1 & 0.47 & 707 & 522 \\
\hline & D & 0.65 & 0.60 & -7 & - & - & - \\
\hline & E & 0.81 & 0.82 & +2 & - & - & - \\
\hline Mean \pm s.d. & & $0.87 \pm 0.12$ & $0.82 \pm 0.11$ & $-5.9 \pm 5.5$ & $0.42 \pm 0.11$ & $999 \pm 286$ & $765 \pm 203$ \\
\hline $0.5 \mathrm{mg} \mathrm{kg}^{-1}$ & A & 0.97 & 0.81 & -17 & 0.51 & 1239 & 871 \\
\hline PET: $n=9$ experiments $/ 5$ animals & A & 0.78 & 0.64 & -18 & 0.23 & 1500 & 1178 \\
\hline \multirow[t]{5}{*}{ MD: $n=9$ experiments $/ 4$ animals } & B & 0.83 & 0.69 & -17 & - & - & - \\
\hline & C & 1.03 & 0.86 & -16 & 0.28 & 1048 & 745 \\
\hline & C & 0.96 & 0.79 & -19 & 0.07 & 1110 & 856 \\
\hline & D & 0.82 & 0.73 & -11 & 0.21 & 1249 & 996 \\
\hline & $\mathrm{E}$ & - & - & - & 0.45 & 550 & 441 \\
\hline Mean \pm s.d. & & $0.93 \pm 0.13$ & $0.79 \pm 0.13$ & $-15.5 \pm 3.8$ & $0.27 \pm 0.14$ & $1320 \pm 432$ & $1004 \pm 375$ \\
\hline $1 \mathrm{mg} \mathrm{kg}^{-1}$ & A & 0.87 & 0.68 & -22 & 0.23 & 1365 & 1027 \\
\hline PET: $n=4$ experiments $/ 3$ animals & A & 0.87 & 0.67 & -24 & 0.11 & 3670 & 2996 \\
\hline \multirow[t]{3}{*}{ MD: $n=5$ experiments $/ 3$ animals } & A & - & - & - & 0.20 & 3200 & 2602 \\
\hline & B & 0.88 & 0.66 & -24 & 0.23 & 1543 & 1241 \\
\hline & D & 0.81 & 0.60 & -26 & 0.38 & 1997 & 1608 \\
\hline Mean \pm s.d. & & $0.86 \pm 0.03$ & $0.65 \pm 0.04$ & $-23.9 \pm 1.7$ & $0.23 \pm 0.10$ & $2355 \pm 1026$ & $1895 \pm 863$ \\
\hline
\end{tabular}

Abbreviations: $\mathrm{AMPH}$, amphetamine; $\mathrm{BP}$, binding potential; $\mathrm{DA}$, dopamine; $\mathrm{MD}$, microdialysis; $\mathrm{PET}$, positron emission tomography.

${ }^{a}$ Mean values of $n=3$ to 4 baseline dopamine samples from -30 to 0 min before amphetamine. 